\title{
CONTEXTE ET TENDANCES DES PROGICIELS DOCUMENTAIRES DANS LES PAYS DU SUD
}

\author{
Ahmed Ksibi \\ Institut Supérieur de Documentation, Tunis
}

\section{Résumé}

Pour la mise à niveau des différents produits et services d'information documentaires (SID) et des bibliothèques des pays du Sud, il est nécessaire d'avoir un système intégré de gestion des bibliothèques (SIGB). Devant l'offre progicielle propriétaire internationalisée et l'irruption de logiciels libres, le choix de logiciels appropriés SIGB est difficile, pour cela il faudrait se doter d'une démarche triptyque :

- s'inscrire dans les alternatives de la coopération qui offre des logiciels et l'accès libre aux ressources. Les logiciels libres deviennent assez proches des principes et pratiques de la bibliothéconomie moderne. Ils ont pour objectif l'accès gratuit et équitable des données, de l'information et du savoir ;

- suivre une méthodologie rationnelle d'analyse des besoins et définir les objectifs prioritaires à moyen et long terme en tenant compte des contraintes techniques dans un cahier des charges pour spécifier les particularités des systèmes informatisés à s'approprier ;

- être informé des dernières tendances des fonctionnalités et des processus documentaires des logiciels.

Les tendances actuelles doivent inclure les systèmes de repérage de l'information multilingue qui tiennent compte de la diversité linguistique et culturelle. D'ailleurs, les instances associatives et normatives de la bibliothéconomie prennent en considération l'élément linguistique dans sa dimension universelle, et æuvrent à réviser l'ensemble des normes, procédures et protocoles dans ce sens.

\section{Abstract \\ Library and Information management software packages for countries from the South: Context and trends}

To upgrade the various products and services of libraries and documentation units in the countries from the South, it is now necessary to turn towards Integrated Library System (ILS ). But the choice of a suitable ILS between «multinational» 
commercial software and open source software becomes very difficult. For this reason we suggest a three steps approach:

- get involved in the alternatives of the co-operation which offers free software and open access to the resources. The free software packages are close to the principles and practices of modern librarianship. They aim at free and equitable access to data, information and knowledge ;

- evaluate the needs rationally and define the top medium and long term priorities, taking into account the technical constraints to specify the characteristics of the computerized systems ;

- stay informed about the new trends in ILS software, including multilingual information systems in order to take into account of the linguistic and cultural diversity.

«Les bibliothèques comme les logiciels libres ont pour objectif l'accès gratuit et équitable des données, de l'information et du savoir »'

\section{Introduction}

Les bibliothèques et les services d'informations documentaires (SID) des pays du $\mathrm{Sud}^{2}$ fonctionnent souvent dans un contexte défavorable et mouvant. Ils subissent souvent les aléas du sous-développent politique, socio-économique et culturel, doublé d'une perception socioprofessionnelle dévalorisante et périmée.

D'aucuns pensent que le dépassement d'une telle situation réside dans l'informatisation et l'emploi des outils et logiciels susceptibles de leurs donner une image reluisante et moderne. Désormais, les logiciels d'automatisation des tâches bibliographiques sont perçus comme un pilier fondamental d'appui aux bibliothécaires. Ils traitent les données sur les documents, les notices, les lecteurs, les budgets et le personnel, etc. Bref, ils sont au cœur des procédures de fonctionnement des bibliothèques et des services de documentation.

En effet, il est actuellement nécessaire d'avoir un système intégré de gestion des bibliothèques (SIGB), ou simplement selon l'abréviation anglo-saxonne un «ILS » Integrated Library Systems, systèmes qui sont devenus des « progiciels » documentaires. Ce ne sont plus des programmes informatiques répondant aux besoins locaux des produits de logiciels, progiciels, qui ont un marché au niveau in-

MORGAN Eric L. Logiciels libres et bibliothèques. In : BiblioAcid, 2004, vol. 1, n 2-3, pp. 1-14.

Pour simplifier une configuration très inégale des pays : les pays en Voie de Développement (PVD) ou les pays du Tiers-monde seront désignés par les pays du Sud comme pays industrialisés ou les Pays développés à économie de marché (PDEM) sont nommés pays du Nord. 
ternational. Parce que l'utilisation de ces progiciels perfectionne les différents produits et services classiques (acquisition, traitement, références, périodiques, prêt, communication) qui seront, après l'informatisation, rationalisés ainsi que largement accélérés. Actuellement, il est admis que l'accélération de la diffusion, par les SID, des produits de l'intelligence humaine passe souvent par l'utilisation de tels outils. De ce fait, ces logiciels doivent exister dans toute bibliothèque, quelle que soit son importance.

Aussi grâce à ces logiciels, les services modernes de numérisation des collections, du réseau et des accès sont maintenant plus faciles à gérer. Ce qui permettrait aux bibliothécaires de mieux consacrer leurs énergies aux fonctions essentielles d'intermédiation et de mieux servir les usagers.

Ainsi la mise à niveau des structures documentaires et des professionnels des pays du sud, qui doit s'opérer pour être au diapason des nouvelles orientations du développement documentaire, exige l'adoption de l'informatique et des logiciels. L'offre progicielle est prolifique et l'irruption des logiciels libres augmente les opportunités d'informatisation, de numérisation et de virtualisation (mise en réseau et sur le Web) des collections et des services. Ce qui pose vraiment problème, ce n'est plus la question du coût exorbitant et de la facture exagérée ${ }^{3}$ de l'acquisition des logiciels, c'est plutôt leurs choix appropriés car leur gamme est très variée et leur production prolifique. Ce qui est devenu essentiel, c'est de connaître l'offre existante du marché des progiciels propriétaires internationalisée et de saisir l'ouverture des publications scientifiques et l'irruption de logiciels libres sur Internet.

Pour les SID des pays du Sud, il suffit de s'inscrire dans de nombreux mouvements coopératifs et collaboratifs venant du Nord et du Sud pour s'approprier des logiciels libres et même propriétaires. Ces mouvements peuvent les mettre à niveau en insérant les Nouvelles Technologies de l'Information de la Communication (NTIC) et par là même juguler les problèmes de gestion notamment par l'implantation des logiciels documentaires les plus adaptées.

Prenant appui sur les réseaux professionnels (l'OCLC Online Computer Library Center, ou le RLG Research Library Group) ou les réseaux de communication globale, en l'occurrence Internet, des mouvances partisanes du libre accès à l'information mettent en oeuvre des formes de collaboration, créant de nouvelles communautés et des nouvelles dynamiques sociales.

Confrontés à une pénurie des ressources documentaires, l'apparition et l'essor des mouvements du libre, du gratuit et de l'Open Access et le développement de la publication scientifique et des archives ouvertes, les professionnels du Sud devraient exploiter de telles opportunités.

L'acquisition du logiciel VTLS pour les Bibliothèques universitaires tunisiennes a coûté autour de 500 milles \$ US 
Pour mieux s'intégrer, il faut connaître le contexte du « capitalisme informationnel », de la complexité et la multiplicité de l'offre (on compte plus que 300 logiciels documentaires ${ }^{4}$ avec un taux de progression important). Les véritables gageures pour le rétablissement et la consolidation des outils et des services des bibliothèques du Sud consistent dans le bon choix des meilleures opportunités du libre et de la gratuité des publications, l'accompagnement des tendances d'ouverture qui cadrent avec les moyens disponibles, les besoins réels et potentiels des usagers.

C'est la maîtrise des flux des connaissances et de l'information scientifique et technique et l'appropriation des NTIC, précisément les logiciels adaptés qui attribuent aux bibliothèques des pays en voie de développement une position de producteur et non de simple consommateur.

\section{Contexte des progiciels documentaires dans les pays du Sud}

Malgré quelques îlots de prospérités, la pauvreté des bibliothèques en Afrique et la précarité de la situation des professionnels dans les pays du Sud, en général, ne font plus de doute. Cette situation a fait l'objet de beaucoup d'études, et d'enquêtes ${ }^{6}$. Celles-ci étalent les lacunes d'une infrastructure, le manque de professionnels, la faiblesse des services offerts...La situation qui prévaut mène à la difficulté de suivre l'évolution des connaissances, conduit à l'empêchement d'accompagner les efforts du développement et entrave la diffusion de l'information.

4 Plusieurs sites listent les logiciels documentaires et de gestion de bibliothèque, à titre d'exemple on peut citer le site de L'Association des Directeurs de Bibliothèques Départementales de Prêt (ADBDP) de France : http://www.adbdp.asso.fr, Ce site compte une soixantaine de systèmes intégrés de gestion de bibliothèques choisis selon des critères précis: le logiciel doit être attesté par une BDP comme étant implanté dans au moins une bibliothèque ouverte au public, le logiciel doit faire l'objet d'une maintenance et qu'il offre les fonctions suivantes :gestion du catalogage et du prêt, interface de recherche pour le public, importation de notices bibliographiques en UNIMARC/ISO 2709.

Dautres sites listent les logiciels libres :

- Framasoft est un annuaire français de logiciels libres

- Logiciels libres pour bibliothèques,

- http://logiciels.bib.free.fr/index.php?pagenews=1

D'autres sites évaluent les logiciels : Étude comparative de logiciels gratuits de gestion de bibliothèque. - F. Dell'Orso, Bibliography Formatting Software: An Evaluation Template, 2007. Voir : http://www.burioni.it/forum/ors-bfs/text/index.html

5 La bibliothèque d'Alexandrie, bibliothèques universitaires en Afrique du Sud, Bibliothèques Nationales de Tunisie et d'Afrique du Sud ...

6 The Status, Reputation and Image of the Library and Information Profession : proceedings of the IFLA Pre-Session Seminar, Delhi, 24-28 Aug. 1992 .ed. by Russell Bowden and Donald Wijasuriya. München : K. G. Saur, 1994. - 228 p. ; 22 cm. - (IFLA Publications ; 68). - ABAD. Enquête sur les bibliothèques publiques au Sénégal. Dakar : Direction du Livre et de la Lecture, 2002 - HELLEMANS Jacques (Dir.) ; TAMBWE Eddie (Dir.).- Bibliothèques, livre, écrit et technologies de l'information et de la communication en République démocratique du Congo : défis et perspectives. 18 ème Colloque international de bibliologie, 27 novembre-3 décembre 2004, Kinshasa. - Paris : L'Harmattan, 2005. - 277 
Dans ces conditions lacunaires, les professionnels doivent être conscients des enjeux de la coopération et du travail collaboratif qui développent les tendances d'ouverture « Open Access, free ». Paradoxalement, les tendances de travail en commun et d'exploitation rationnelle des ressources humaines matérielles et professionnelles ont été inaugurées dans les pays qui disposent le plus de moyens, qui normalement peuvent se passer des apports d'autrui. Les réseaux coopératifs de bibliothèques, l'OCLC ou le RLG, qui ont fonctionné depuis les années soixante dix au USA, sont devenus avec leurs catalogues collectifs des énormes réservoirs de données bibliographiques ${ }^{7}$. Les associations telles que la « Free Software Fondation» de développement des logiciels open source agissent avec une logique proche de la communication du monde universitaire, où le profit ne compte pas et où prédominent l'échange des données et la gratuité d'accès. Tous ces réseaux qui stimulent le partage des coûts et des ressources, l'échange et la gratuité, ont été développés dans des institutions des pays riches et notamment les bibliothèques riches.

Ces mouvements d'ouverture, prônant « l'appropriation citoyenne » des connaissances et de l'informatique, permettent de s'affranchir, un tant soit peu, de la dépendance vis-à-vis des grandes multinationales. Ces mouvements tentent de contrer les stratégies de « verrouillage » du capitalisme informationnel qui tente de briser l'essor du libre par le biais d'actions judiciaires et par la volonté d'imposer la protection via les brevets.

Les accords relatifs aux services commerciaux d'information et à la propriété intellectuelle, dans le cadre de des organisations néo-libérales : l'Organisation mondiale du commerce (OMC) et de l'Organisation Mondiale de la Propriété Intellectuelle (OMPI), vont accabler encore plus le libre accès à l'information et aux connaissances ${ }^{8}$.

Face à « la fermeture » des accès aux connaissances, les alternatives s'articulent autour des modèles de la coopération, du travail collaboratif et des nouveaux dispositifs du droit à l'accès à l'information'. L'ouverture devient le modèle d'action privilégié pour l'amélioration des produits et services des bibliothèques et des unités documentaires du Sud.

7 L'OCLC contient des renseignements (70 millions de références) sur les collections variées de 54,000 bibliothèques de plus 100 pays

8 L'effet des monopoles de propriété, se trouve amplifié lorsque ce monopole s'applique aux ressources informationnelles. L'avenir de l'ensemble des bibliothèques pourrait être menacé par une série de traités commerciaux internationaux. Voir les positions de l'IFLA sur les négociations du Traité de l'OMC notamment sur l'Accord sur les aspects des droits de propriété intellectuelle liés au commerce «TRIPS ». Un déséquilibre règne entre l'obligation de la protection des droits des producteurs, et ceux des utilisateurs de l'information

9 Le Creative Commons et le "copyleft" favorisent la disponibilité sur Internet de ressources cognitives en accès libre. 
La coopération internationale, dans un cadre libéré d'une dépendance postcoloniale, intègre plusieurs actions de dons et d'échanges entre professionnels à tous les niveaux: équipements, collections, contenu, contenant et systèmes de gestion de bibliothèque. $^{10}$

Lorsque les infrastructures existent et qu'elles sont fonctionnelles, l'accès au réseau Internet représente une source d'information importante sur les produits et œuvres collaboratifs et gratuits. Avec des connaissances et des compétences en informatique et des données actualisées sur les nouveautés du marché des progiciels, le choix pourrait être plus intelligent et plus intelligible. De même, avec plus d'information et une meilleure connaissance des réseaux et des institutions à contacter, il est possible de bénéficier de dons « croisés » d'équipements, de collections et de ressources provenant de différents organismes gouvernementaux (OIF, UNES$\mathrm{CO}$ ) et non gouvernementaux (IFLA).

Les logiciels libres ILS, de la même manière que les périodiques en « open access » sur le réseau, devraient permettre d'améliorer les conditions des SID du Sud. Ces dernières années, les groupes et génies des logiciels libres se sont amplifiés (une croissance annuelle de $30 \%$ du libre). Ils peuvent contribuer, à coûts réduits, à un meilleur fonctionnement des SID du Sud. ${ }^{11}$

Les SIGB utilisables en réseau et incluant les principales fonctionnalités de la chaîne documentaire, apparaissent de plus en plus indispensables. Ces outils de gestion qui pourraient non seulement exploiter avec un maximum d'efficacité les collections et la documentation dont les bibliothèques disposent, mais aussi permettent aux bibliothécaires de s'acquitter convenablement des tâches administratives (gestion du budget, rapports statistiques des services...) et les services de référence pour leurs usagers.

\subsection{Les logiciels propriétaires documentaires \& la marchandisation de l'information}

Cependant les logiciels ILS performants sont majoritairement détenus dans les pays du Nord par des compagnies de l'industrie du logiciel et des firmes commerciales qui ne s'intéressent qu'à maximiser les profits. Celles-ci offrent un ensemble large de produits diversifiés de logiciels propriétaires. L’hégémonie des ILS logiciels pro-

10 L'Organisation des Nations Unies pour l'Education, la Science et la Culture (UNESCO) est l'exemple type de l'organisation consacra un Programme général d'information (PGI).L'essentiel de ses programmes renforcent les capacités des pays en développement, dans les domaines de l'information et de l'informatique en particulier par le développement et la diffusion en coopération des logiciels CDS/ISIS et IDAMS. Elle a été relayée par les réseaux régionaux (RINAF, RINSCA, RINSEAP, par exemple) des organisations régionales : ALECSO , ISESCO ...

11 Association africaine des utilisateurs de logiciels libres - Association francophone des utilisateurs de Linux et des logiciels libres (AFUL) 
priétaires Américains (plutôt Etats Uniens) des SIGB performants (Unicorn, Horizon, VTLS, Follett ...) a été réconfortée par la vague d'informatisation des deux dernières décennies dans les bibliothèques des pays du Nord. Selon les sources les plus fiables ${ }^{12}$, le marché des SIGB se fait pour l'essentiel avec des logiciels propriétaires des Etats Unis (les logiciels du Follett Software Company ${ }^{13}$ sont présents sur à peu près 40 mille sites aux Etats Unis, occupant $60 \%$ du marché Américain des bibliothèques scolaires, Horizon et Unicorn dans les Bibliothèques Universitaires BU ...). D'après l'étude sur l'équipement des BU en ILS, menée par l'Education nationale Française en $2002^{14}$, ce sont les produits américains qui prédominent.

C'est ainsi que le marché ${ }^{15}$ des ILS, malgré son étroitesse, participe à la logique du « capitalisme informationnel » Américain et obéit à ses lois implacables. En étudiant les activités de ses compagnies, on se rend vite compte que les firmes dominantes ont les transactions habituelles du capitalisme : c'est le capital qui dicte le rachat des petites compagnies, la création de nouvelles firmes et fusion entre cellesci, ce n'est pas l'intérêt des concepteurs et des usagers qui est le plus important. Par exemple, on a assisté en 2006 à la domination de Follett Software Company (FSC), qui a phagocyté de petites sociétés comme archrival Sagebrush et Extensity. Dans la même année, la société Dynix ${ }^{16}$, propriétaire du logiciel Horizon, a été achetée par sa concurrente Sirsi, propriétaire de l'Unicorn ${ }^{17}$, elle a créé un nouveau ILS plus performant (ROM). Dans le même contexte, la concentration est aussi remarquable pour le marché des périodiques électroniques dans lequel le nombre d'éditeurs est de plus en plus réduit dans la mesure où les petits éditeurs sont rachetés par les gros.

Il s'agit de la même devise du capitalisme : maximiser la production, booster la consommation afin d'accumuler toujours plus de capitaux et de pouvoirs. Celui-ci place les pays du Sud en situation de dépendance totale pour la maintenance des logiciels et pour l'accès à l'information.

Les projets d'informatisation des grandes bibliothèques au $\operatorname{Sud}^{18}$ qui sont financés par les organismes du néo-libéralisme, la Banque Mondiale par exemple,

12 SOURCE: Library Journal AUTOMATED SYSTEM MARKETPLACE 2007, Aussi les études de TOSCA publiées dans : Livres Hebdo ; Archimag

13 Follett avec ses logiciels destinés pour les collèges : Follett Circulation Plus, Catalog Plus Winnebago Spectrum.

14 Sources : http://www.sup.adc.education.fr/bib/Acti/Infor/infor.htm

15 Selon l'évaluation Marshall Breeding les revenus totaux d'industrie des logiciels SIGB en 2006 étaient environ 570 million \$ US en 2005 étaient environ 535 millions\$, en hausse d'environ 6\% par rapport à l'estimation révisée de l'auteur de505 millions \$ en 2004.

16 Breeding, Marshall. Reshuffling the Deck. Library Journal; 4/1/2006, Vol. 131 Issue 6, p. 40-54.

17 Idem "Ex Libris, and Endeavor was merged into Ex Libris. Follett Software Company acquired archrival Sagebrush, and Extensity morphed into Infor Library Solutions."

18 Sohier Ibrahim. Saleh. Electronic Library Systems and their Role in Providing Developed Technological Services: Requirements and standards. In: The proceedings of the AFLI $12^{\text {th }}$ conference. pp. 275-288. 
s'orientent généralement vers ces logiciels propriétaires Etats Uniens. Les prix sont souvent prohibitifs tant pour l'achat des licences de ces logiciels propriétaires que pour le reste du matériel et des abonnements aux bases de données... Ainsi, les coûts des abonnements aux périodiques scientifiques qui ont, selon Morgan ${ }^{19}$, « augmenté de manière bien plus rapide que l'inflation (les prix étant désormais totalement hors de contrôle), de moins en moins de bibliothèques (et donc de moins en moins de personnes), même dans les pays du Nord, ont accès à ces publications. »

Ce constat de la situation du « capitalisme informationnel » et des lacunes de la documentation, que les bibliothèques du Sud vivent ne veut pas dire qu'elles sont dans une situation d'aporie. Ceci ne doit nous amener à penser qu'elles sont dans un chemin sans issue, et ne doit pas non plus inciter au pessimisme.

Au contraire, une fois le contexte connu et bien posé, il faudrait simplement être informé, et se doter d'une démarche triptyque pour un meilleur accès aux connaissances:

- s'inscrire dans les alternatives de la coopération qui offre des logiciels et qui permet l'accès libre aux ressources ;

- suivre une méthodologie rationnelle d'analyse des besoins et définir les objectifs prioritaires à moyen et long terme en tenant compte des contraintes techniques dans des documents de référence « un cahier des charge » pour spécifier les particularités des systèmes informatisés à s'approprier ;

- être informé des dernières tendances des fonctionnalités et des processus documentaires des ILS.

\section{Alternatives de la coopération d'accès libre aux sources}

De nombreux mouvements de solidarité et de partenariat venant du Nord et du Sud, visent le partage des ressources informationnelles pour un meilleur accès aux connaissances. C'est un défi majeur pour les bibliothèques du Sud que d'établir le canevas de liaisons nécessaires pour s'y inscrire.

Les effets des relations de coopération, de partenariat et d'intégration des ressources informationnelles sont assez visibles dans le domaine de l'informatisation des bibliothèques depuis les années soixante, comme l'exemple précédemment cité de OCLC. Elles se sont incrustées et se sont enracinées par la suite dans le paysage documentaire du Sud. Ainsi depuis la fin des années quatre vingt, des logiciels documentaires sont délivrés gratuitement, à large échelle, par la coopération bilatérale et multilatérale. Les plus connus sont les ILS de la famille des systèmes d'information

19 Eric L. Morgan. logiciels libres et bibliothèques .- In BiblioAcid Volume 1, Numéro 2-3, Mai-Juillet 2004 - http://www.nicolasmorin.com/BiblioAcid revue/BAv1n23.pdf 
intégrés ISIS (Integrated Set for Information System), ensemble intégré de systèmes d'information développés en 1965, puis ils sont relayés par le logiciel MINISIS, conçu pour des mini ordinateurs gracieusement fournis par la coopération canadienne à travers son Centre de Recherche pour le Développement International (CRDI). Depuis les années quatre vingt, la coopération internationale de l'UNESCO, a développé et diffusé gratuitement le célèbre logiciel CDS/ISIS pour des micros ordinateurs.

Aussi l'accès à l'information scientifique à travers des bases de données et des publications pour les bibliothèques du Sud est fourni gratuitement par plusieurs organismes. Les programmes d'échange d'information établis par l'Agricultural Information System (AGRIS) du FAO et actuellement par d'autres institutions ${ }^{20}$ permettent d'estimer des effets bénéfiques de la coopération sur l'accès libre aux connaissances dans des domaines vitaux pour le tiers monde.

Ce qui est plus rare au Sud, c'est l'appropriation de ces technologies qui cherche à donner aux pays en voie de développement une véritable autonomie. Toutefois, quelques bibliothèques des pays du Sud se sont dotées des logiciels « maison » assez performants : le logiciel Alice ${ }^{21}$ qui fonctionne dans les bibliothèques publiques en Egypte, l'expérience réussie du Système Normalisé de Gestion De Bibliothèque (SYNGEB) qui fonctionne sur le Réseau Inter-Bibliothèques Universitaires (RIBU) en Algérie. Cet ILS gère un catalogue collectif des fonds important (soit plus d'un million de documents) ${ }^{22}$ mis en ligne des 10 bibliothèques membres.

\subsection{Les logiciels libres versus les logiciels propriétaires}

Sur ce dernier point, une appropriation plus actuelle des NTIC est entraînée par l'emploi des logiciels non pas propriétaires mais des logiciels libres et les programmes informatiques Open source. L'énorme avantage de concession de ceux-ci réside dans la possibilité de changer le statut de l'utilisateur de ces logiciels en partenaire si des développements spécifiques sont assurés. Par la disponibilité du code source, les possibilités d'intervention des partenaires sur le logiciel à travers plusieurs apports. La création ou l'amélioration de fonctionnalités, les développements permettent d'atteindre un niveau d'autonomie élevé et d'assurer un véritable transfert de technologies.

Avec les logiciels libres, l'aspect commercial va s'atténuer pour se transformer en relation de partenariat. Le lien avec la société créatrice du logiciel n'est pas obligatoire puisqu'une bibliothèque peut se réserver la liberté de choisir son prestataire de service.

\footnotetext{
20 L'Open Society Institute (OSI) a engagé le projet eIFL.

21 Différent du logiciel Alice. Système de gestion de bibliothèque Alice, développé et distribué par Softlink en France.

22 La Lettre d'EchosDoc n 1 (1er juin 2007), www.echosdoc.net
} 
Par contre, les logiciels documentaires propriétaires sont régis par des relations de type traditionnel entre fournisseurs/clients. L'utilisateur est donc souvent lié par des contrats fermes, il se trouve parfois asservi par des clauses en faveur du vendeur.

Pour les pays du Sud, maîtriser ces logiciels libres revient en partie à maîtriser leur développement et le transfert d'information pourrait présenter une alternative intéressante aux logiciels propriétaires. De plus, les fonctionnalités des SIGB gratuits se rapprochent petit à petit de leurs grands frères commerciaux. On peut aujourd'hui envisager l'informatisation d'une bibliothèque de bonne taille, avec des produits comme Koha ou PMB. C'est pour cela qu' on assiste, selon Marshall Breeding, à une croissance de $30 \%$ du libre, ces dernières années. ${ }^{23}$ L'année 2006 est l'année pendant laquelle les logiciels libres ont acquis une présence notable dans le domaine documentaire. D'ailleurs avec la migration des bibliothèques vers le libre accès, les compagnies de programmes propriétaires dominants redoutent sa concurrence.

Pour nuancer, il ne faut pas avoir des illusions. Les logiciels libres qui ne sont, en fait, ni tout à fait libres, ni tout à fait gratuits. De prime abord, il faut avoir une licence ${ }^{24}$ parfois contraignante. La liberté suppose la responsabilité des professionnels et des institutions qui ont la licence et le programme ouverts. Ils doivent forcément respecter l'esprit coopératif et le partage volontaire des connaissances pour faire profiter toute la communauté des usagers de l'expérience acquise. La contribution est multiple : celle de redistribuer des copies, celle d'améliorer le programme et de communiquer ses améliorations... L'esprit de partage permet de revenir au modèle d'échange académique ou des pionniers de la programmation ${ }^{25}$ du premier temps de l'informatique. Au début, les logiciels écrits en langage informatique étaient destinés à être partagés avec les autres, tout comme les résultats de la recherche scientifique qui étaient partagés par le biais des publications scientifiques.

Les pionniers du développement des logiciels, programmeurs et analystes, et les universitaires n'hésitaient pas à publier leur code source, pour stimuler la relecture et la validation par les pairs avec toute la rigueur qui s'impose. ${ }^{26}$ Les fabricants d'ordinateurs, de leur côté, encourageaient, au début une telle attitude (IBM, par exemple). Ce n'est qu'au début des années 80 , que cet esprit de partage se trouve ré-

23 Marshall Breeding. Private equity moves into the ILS, and open source support emerges, in : Library Journal, 4/1/2007. - http://www.libraryjournal.com/article/CA6429251.html?q=Market+place+ april+2007

Les plus connues sont les licences GPL (GNU Public licence) et la licence de type BSD (Berkeley)

25 Par exemple, en 1975 la contribution du jeune Bill Gates à un nombre de programmes écrits en BASIC qui fonctionnaient gratuitement sur le micro ordinateur ALTAIR 8800.

26 L'informaticien Richard Stallman, qui travaillait pour le MIT dans un environnement où les logiciels étaient partagés, développa en 1984 le concept du logiciel libre, créa la "Free Software Fondation", le projet de développement de logiciels libres (GNU). 
cupéré par le capitalisme financier qui investissait lourdement dans l'industrie du logiciel. Celui-ci devenait «propriétaire », et imposait l'acquisition de la « licence d'utilisation ». La non-publication du code source, interdit à l'utilisateur d'intervenir sur le logiciel pour l'adapter à ses besoins propres et il est obligé d'attendre que de nouvelles versions soient mises sur le marché.

\subsection{Logiciel libre et gratuiciels}

Un logiciel libre, qui s'achète différemment des logiciels commerciaux, n'est pas forcément sans charges et de nombreux coûts sont cachés. Comme le précise Mor$\operatorname{gan}^{27}:$ « le terme « libre » doit être compris au sens du mot latin liberat, c'est-à-dire libérer, et pas nécessairement au sens de « gratis », c'est-à-dire sans contrepartie. ${ }^{28}$

Un logiciel libre est considéré gratuit quand la licence n'a été payée qu'une fois, ce qui correspond en particulier à l'investissement public qui considère le logiciel acquis comme un équipement qui ne s'achète qu'une seule fois.

Dans le cas des gratuiciels, la licence est gratuite. Mais une fois le logiciel donné ou téléchargé, il faut avoir une certaine maîtrise de l'informatique sinon beaucoup de charges sont à supporter. Les charges concernent évidemment les coûts des compétences humaines nécessaires pour assurer l'installation, le paramétrage, la gestion et la formation, qui sont souvent difficiles à détenir. Pour de nombreuses institutions, qui n'ont pas de service informatique, telle que la majorité des bibliothèques, la maintenance du logiciel libre peut coûter beaucoup d'argent et de temps. Ces charges ne sont en principe pas rencontrées chez les bibliothèques qui suivent les éditeurs de progiciels commerciaux. Ceux ci sont toujours prêts à vendre un « développement spécifique » et de « costumériser» leurs progiciels.

Avec une mentalité inextricable d'éternel assisté, certains bibliothécaires des pays du Sud attendent souvent des solutions, clés en mains, immédiatement opérationnelles et pérennes. Or par définition, les logiciels libres sont en constante évolution et en réadaptation. Ils ne sont jamais finis car ils se sont développés pour des besoins spécifiques d'une bibliothèque. Les mises à jour sont à faire soi-même ou à travers des sociétés de services qui assurent le support du produit.

L'essor des nouvelles Sociétés de Services de Logiciels Libres SSLL : genre : C'est le cas de LibLime aux Etats Unis, PMB Services allouées au soutien du logiciel PMB, Inéo Media system en France, spécialisées dans le support des bibliothèques utilisant le logiciel libre Koha originaire de Nouvelle-Zélande. Ces sociétés SSLL peuvent prospérer en rendant des services (installation, développements spécifiques, maintenance) autour de logiciels gratuits construits avec des technologies

MORGAN Eric L. Op.Cit.

28 Si le terme « free » en anglais est ambigu, le français quant à lui marque bien la distinction entre « libre » et « gratuit» 
libres. Les concepteurs et les sociétés de services seront récupérés, malgré la bonne volonté et les bonnes intentions, par le capitalisme financier. Ceci est démontré par les nouvelles sociétés du libre qui sont cotés en bourses et les tendances du marché international évoluant ces dernières années vers le libre. ${ }^{29}$

Malgré notre penchant vers le libre, dans le sens du partage et de la transparence, les choix en ce domaine ne sont pas nécessairement du « tout commercial » ou du « tout libre ». La possibilité de fonctionner correctement et d'une manière performante est déterminée par le choix du logiciel correspondant aux besoins de la bibliothèque à ré-informatiser, de l'établissement et de l'environnement dans lequel elle fonctionne.

\section{Une méthodologie correcte et des documents de référence : étude de faisabilité, cahier des charges}

Le processus de sélection et d'évaluation des logiciels passe par une méthodologie de diagnostic des sources d'information, des procédures et des projections des systèmes d'information à implanter. Ceci est un préalable nécessaire à la détermination des modalités de fonctionnement pour l'amélioration des systèmes d'information.

L'informatisation, la réinformatisation, la numérisation, la virtualisation ne parviennent à atteindre leurs objectifs avec succès, que par une démarche rationnelle qui doit être consignée dans des documents de référence. Une étude de faisabilité, un cahier des charges, des manuels de gestion et de maintenance, l'élaboration de ces documents se résume en deux prospections communes à toutes les études de ce genre:

- Diagnostic de fonctionnement de chaque bibliothèque et service de documentation et d'information;

- Conception d'un modèle de fonctionnement et d'organisation du système d'information projeté.

Comme nous l'avons précisé plus tôt, acquérir un logiciel commercial performant, est généralement très coûteux. Il faut passer par ces études qui permettent de mieux voir le bien fondé de cet achat et de faire une comparaison avec des produits semblables dans le monde des logiciels libres.

Pour des institutions et des SID du secteur public, en particulier, il faut rédiger un cahier des charges ${ }^{30}$, qui est une étude exprimant les besoins de façon détaillée. Il s'agit d'établir les contraintes techniques et administratives, respectivement, dans

29 Marshall Breeding. Op.Cit.

30 L'Association des directeurs de bibliothèques départementales de prêt (ADBDP) a publié sur son site un modèle de « Cahier des charges pour l'informatisation de bibliothèques en réseau ». 
des clauses techniques et des clauses administratives. À ce document contraignant, les soumissionnaires vont répondre par des offres après la diffusion d'un appel ou d'une commande pour les fournisseurs, dans les média, au niveau national ou international.

L'élaboration des études : Etude de Faisabilité EF, Cahier des Charges CC, permet d'avoir un dossier d'appel d'offres. Ce dossier comporte une définition du marché et des prestations demandées. Le marché peut être segmenté en postes ou regroupé en un seul achat. Exemple Premier poste : l'acquisition d'un système de gestion de bibliothèques et de documentation/Deuxième poste : l'acquisition du matériel nécessaire à la mise en exploitation du système/Troisième poste : la fourniture de service de traitement et d'analyse documentaire.

Ce dossier n'est nécessaire que pour les commandes publiques dont le montant est supérieur à une enveloppe financière préalablement fixée par des textes officiels. ${ }^{31}$ Ces textes législatifs ou réglementaires, qui varient selon les pays et la nature du marché ${ }^{32}$, régissent les principes de passation des marchés publics pour les entreprises et établissements du secteur public. Les acquisitions au-dessous d'un certain montant ne nécessitent pas l'élaboration d'un CC et un dossier d'appel d'offres. Toutefois l'étude et la méthodologie sont une obligation pour les bibliothèques qui n'ont pas de moyens et qui désirent rationaliser à la fois leurs acquisitions et leurs travaux techniques et administratifs.

Même si le choix s'est porté vers les logiciels libres, l'étude préalable est d'autant plus nécessaire que la rationalisation des procédures et des décisions manque dans les bibliothèques du Sud. Le management et la gestion sollicitent des études consignées. Ces études auront, au moins, pour objectifs d'inventorier les principaux gratuiciels de gestion de bibliothèque sur la base des fonctionnalités annoncées. Plusieurs sites ${ }^{33}$ actualisés facilitent la recherche des éléments de comparaison plausibles. Le premier critère de sélection des logiciels libres est l'adéquation de ses fonctionnalités par rapport aux besoins des bibliothèques. Certains logiciels seront éliminés, dès cette première analyse de besoins car ils se révèlent inadaptés. La possibilité d'adaptabilité et du déploiement aisé des logiciels dans un environnement ne comportant pas de ressources humaines importantes en matière informatique sont appréciables. La facilité de leurs procédures d'implantation, l'ergonomie ainsi que les commodités de paramétrage sont autant de critères à mettre en exergue. D'autres

31 Un appel d'offre dans le marché public l'Etat Français il faut plus de 130.000 euros ou 200.000 euros pour les collectivités locales, l'enveloppe nécessaire pour passer par l'appel d'offre est moindre en Tunisie, il faut 40000 dinars $=23500$ euros pour établir un appel d'offre les études et la fourniture de biens ou de services dans le secteur de l'informatique et des technologies de la communication. Code portant réglementation des marchés publics issu du décret du 7 mars 2001 en France, correspondant au décret du 17 décembre 2002 en Tunisie, tel que modifié et complété par le décret $\mathrm{n}^{\circ} 2003$ 1638 du 4 Août 2003 et du décret $n^{\circ}$ 2004-2551 du 2 novembre 2004. 
critères non moins importants concernent leurs fonctionnalités. En particulier leur praticabilité de fonctionner adéquatement en réseau, l'efficacité réelle des procédures bibliothéconomiques documentaires respectives, la stabilité, la sécurité et l'opérationnalité de la récupération de données existantes.

\subsection{L'évaluation des logiciels}

Après l'inventaire des logiciels, les modalités d'analyse des solutions logicielles devront être suivies pour obtenir l 'intégralité des informations requises. Dans cette phase, il s'agit d'élaborer un cadre de test. Le canevas est généralement sous forme de grille qui rassemble, de façon organisée, les éléments jugés indispensables et les fonctionnalités souhaitables. Ainsi, pour les critères retenus, une note est attribuée et une pondération est introduite par rapport aux besoins spécifiques des bibliothèques.

Différentes modalités d'analyse des offres se font selon une grille d'ensemble figurant, généralement, dans le cahier des clauses techniques. Les critères d'analyse figurant dans la grille doivent être univoques et objectifs, Le tableau suivant décrit une activité documentaire qui forme un exemple d'un module de la grille:

\begin{tabular}{|l|l|l|l|l|l|}
\hline $\begin{array}{l}\text { Fonctions : } \\
\text { Gestion des } \\
\text { collections }\end{array}$ & Remarques - Questions & Obl & Souh & Rép \\
\hline Inventaire & $\begin{array}{l}\text { Le logiciel doit gérer les inventaires des } \\
\text { documents de la bibliothèque. Il est pos- } \\
\text { sible d'éditer un inventaire annuel des } \\
\text { collections avec les entrées et sorties ; } \\
\text { cet inventaire est valorisé. }\end{array}$ & $\mathrm{X}$ & & & \\
\hline Récolement & $\begin{array}{l}\text { Le logiciel doit gérer les recollements, à } \\
\text { partir d'un tri topographique dans le ca- } \\
\text { talogue. }\end{array}$ & $\mathrm{X}$ & & \\
\hline
\end{tabular}

Les 2 premières colonnes indiquent les fonctionnalités requises du logiciel. Ainsi pour l'activité de gestion des collections, la grille décline 2 différentes fonctionnalités, clairement définies à faire exécuter par le programme. Les 3 colonnes suivantes indiquent le degré d'importance de la fonction: $\mathrm{Obl}=$ Obligatoire/Imp = Important/ Souh. $=$ Souhaitable . La dernière colonne $«$ Rép $»$ doit être obligatoirement remplie par les soumissionnaires. Il est possible d'y répondre par oui ou par non « OUI » indique que la fonctionnalité est présente, disponible et fonctionnelle dans l'application et qu' elle est comprise dans l'offre du soumissionnaire. «NON » indique que la fonctionnalité n'est pas présente dans l'application. L'absence totale de réponse équivaudra à un « NON ». 
La grille définitive devrait recueillir, de façon organisée les éléments jugés indispensables pour le meilleur fonctionnement du système d'information projeté. Reste que beaucoup de questions, comme la facilité de l'utilisation ou l'ergonomie comportent des jugements subjectifs difficilement mesurables, font exception à cette grille. Pour cela la comparaison des offres se fait selon des procédures d'évaluation différentes selon l'importance de l'acquisition. Une organisation sous forme de conseil est requise. Par exemple une réunion d'une équipe d'experts ${ }^{34}$ jugeant, à l'aide de critères objectifs (quantitatifs et qualitatifs), les offres disponibles qui deviennent pléthoriques avec la diffusion par Internet.

Des compléments de procédures d'évaluation utilisent aussi le contact direct ; les discussions avec les fournisseurs devraient être bannies au début de l'étude. La stratégie et les techniques du Marketing des sociétés informatiques sont plus développées que les perspectives de discernement des bibliothécaires.

Des lectures attentives de la documentation technique fournie par les éditeurs de logiciels et de documents d'aide détaillés suffisent pour réunir des éléments d'appréciation. Toutefois, lorsque les logiciels ont une fonctionnalité qui n'est pas développée, et posent des problèmes, le contact avec les éditeurs des logiciels peut amener des démonstrations, à la demande. Les démos et les tests présentent, en général, l'inconvénient d'interroger une base parfaitement travaillée, et n'exposent pas les cas dans des conditions de travail normales. La consultation des forums et des listes de discussion ${ }^{35}$, et le recours aux témoignages de bibliothécaires utilisant ces produits pourraient être un complément d'information appréciable. La possibilité de porter des appréciations ou de compléter l'évaluation par des questions ouvertes et une colonne des « commentaires », épargne, par ailleurs, des critères subjectifs à la grille. Celle-ci doit comporter toutes les activités à automatiser, la panoplie devenant de plus en plus large jusqu'à prendre presque toutes les activités exercées par le bibliothécaire avec une dextérité, qui parfois le dépasse.

\section{Les spécifications fonctionnelles des progiciels documentaires}

Les SIGB sont aujourd'hui plus que jamais intégrés, portant sur l'ensemble des aspects bibliothéconomiques et les activités administratives des SID. Les SIGB sont généralement regroupées en différents modules nécessaires au fonctionnement du système d'information selon le mode numérique. Qu'ils soient organisés en un ou

34 Plusieurs formules d'organisation et de structuration doit être définies : Cellule, Commission, Comité de Pilotage

35 Logiciels bibliographiques/documentaires : évaluation « Bibliography Formatting Software: An Evaluation Template ». www.burioni.it/forum/ 
plusieurs modules, les SIGB doivent assurer au moins les principales fonctions bibliothéconomiques. Les activités peuvent être subdivisées en deux volets:

- Une gestion administrative (personnel, budget, tableaux de bord...) et l'édition de statistiques pour l'évaluation et pour chacun des modules bibliothéconomiques. Cette gestion peut être définie selon la nature des ressources disponibles, par exemple les ressources humaines et informatiques à informatiser concernent :

La gestion des personnes et des institutions, du personnel, des fournisseurs, des abonnés;

Les activités de la maintenance du logiciel : les versions du SIGB et le niveau de son développement, la structure de la base, l'ergonomie, le mode de fonctionnement, les normes de codage, le système d'exploitation, l'aide et la sécurité

Les activités de gestion du réseau (le serveur, la gestion des droits et profils, les postes clients, l'OPAC, l'importation, l'exportation,) les navigateurs sécurisés sur les postes multimédia publics, les logiciels des firewalls et du routeur, Le logiciel de comptage du temps de connexion sur les bases payantes sur les postes publics.

La gestion d'autres ressources (ressources financières et matérielles) peut être définie et comporte nécessairement l'analyse des tâches, des données et des procédures de chaque fonction.

- Une gestion des activités techniques de la chaîne documentaire peut être mentionnée successivement:

La gestion des acquisitions;

La gestion des périodiques;

Le catalogage (et notamment les normes de catalogage, les bordereaux de saisie et la gestion des autorités);

L'indexation;

La recherche documentaire;

Les éditions;

La gestion des prêts et de la circulation (avec le paramétrage de profils d'abonnés, la durée du prêt, la réservation, les relances, les pénalités, ...etc).

Ces fonctionnalités figurent comme modules dans, pratiquement, tous les anciens SIGB connus, nécessairement pour être au diapason il faut scruter les nouvelles fonctionnalités. Se lancer dans le choix des logiciels gratuits ne doit pas être une aventure sans lendemain. Pour cela il faut une mise à jour des connaissances, concernant les fonctionnalités potentielles des logiciels, et il faut des capacités pour explorer les tendances, sur les quelques années à venir, pour avoir un logiciel adapté aux changements. 


\subsection{Tendances des fonctionnalités des SIGB}

Avec les progrès vertigineux des technologies du réseau, notamment à travers les catalogues mis en ligne OPACs au service des usagers, les bibliothèques sont de plus en plus présentes sur le Web proposant des références et des contenus dans des langues diverses et desservant des usagers de différents pays.

L'avancée du codage des caractères dans les systèmes informatiques, a permis l'accès multilingue à l'information documentaire. Les SIGB ont surmonté les problèmes relatifs à l'enregistrement des données en plusieurs graphies, qui jusque là représentait un handicap de taille, non seulement pour les SID du Sud dont l'alphabet est non latin, mais aussi pour les grandes bibliothèques desservant un public multilingue notamment dans les pays européens bilingues et multilingues. Les SIGB les plus appropriés aux SID du Sud seront forcément multilingues pour évoluer dans un environnement documentaire international et pour répondre aux besoins spécifiques des usagers des bibliothèques et des centres de documentation en matière de recherche de l'information multilingue.

Parmi les tendances des fonctionnalités à connaître actuellement, il s'agit dans cette note d'analyser les principales : Les technologies ouvertes, la conformité aux normes professionnelles, les interfaces et les portails web avec les traitements multilingues. Les spécifications techniques des principales fonctionnalités devraient s'insérer comme des critères dans les cahiers des charges pour les SIGB à acquérir.

Les trois spécifications à inclure dans le choix des logiciels, sont ci-dessous sommairement décrites :

\subsubsection{Technologies ouvertes}

Les logiciels SIGB doivent nécessairement être basés sur des technologies ouvertes (PHP, MySQL,...) qui sont actuellement largement répandues. Surtout que ces technologies remontent graduellement du serveur vers les postes de travail bureautique (OpenOffice.org, Firefox, et les autres logiciels de Mozilla project : Thunderbird, Nvu,...). Les systèmes d'exploitation «portables » comme Unix, Linux (KDE et Gnome), indépendants du matériel, permettent de développer facilement de nouvelles fonctions puisque les programmes sources sont disponibles.

\subsubsection{Normes et standards}

Les logiciels SIGB doivent respecter les normes professionnelles, essentiellement les formats de catalogage connus :

- Le SIGB doit pouvoir gérer un système bibliographique en format de type UNIMARC qui doit être aisément paramétrable, en fonction de chaque bibliothèque et du type de documents gérés par le système (monographies, publications en séries, etc.); 
- Le SIGB doit pouvoir exporter et importer des informations notamment des notices bibliographiques et d'autorités en format UNIMARC;

- Le SIGB doit pouvoir charger des fichiers bibliographiques en format ISO 2709 ou en format texte structuré à partir de n'importe quel support informatique : bandes, disquettes, CD-ROM, DVD,...

En plus des normes professionnelles les SIGB doivent être compatibles avec des normes informatiques destinées à favoriser l'échange des données :

- ISO 23950 (ANSI Z39.50) : protocole de communication client-serveur qui permet l'interrogation via un réseau informatique de bases de données réparties et hétérogènes. Ce protocole est essentiellement utilisé dans le monde des bibliothèques, il permet la recherche fédérée, autrement dit, la possibilité d'interroger plusieurs catalogues simultanément ;

- SRU/SRW est l'acronyme pour (Search/Retrieve via URL et search/Retrieve Web service). Ces deux protocoles sont les successeurs du protocole Z 39.50 et en « constituent une évolution et ce, vers les technologies web, via les webservices $\gg .{ }^{36}$ Ils sont destinés à faciliter la recherche sur le net et ont été développés initialement pour les besoins de la Bibliothèque du Congrès qui en assure la maintenance. Le SRU est bâti sur le langage standard de syntaxe de requête CQL (Common Query Language) qui lui permet de spécifier la syntaxe des requêtes et celles des réponses.

- UNICODE : standard informatique développé par le Consortium UNICODE (http://www.unicode.org/), qui permet de coder tous les jeux de caractères de façon unique. La conformité au standard Unicode est maintenant nécessaire car il fournit « une représentation non ambiguë de texte à travers une gamme de manuscrits, de langues et de plateformes.» ${ }^{37}$

\subsection{3 les interfaces et les portails du web et le multilinguisme :}

Avec le développement vertigineux du web, l'omniprésence de ses navigateurs et l'évolution du format XML, une migration du monde du savoir vers l'Internet et ses protocoles est manifeste. Les bibliothèques migrent, indubitablement et délibérément, vers le mode virtuel.

Pour cela le SIGB doit pouvoir fonctionner en modèle client/serveur et supporter le protocole TCP/ IP. Tous les modules du SIGB doivent pouvoir fonctionner en réseau avec des systèmes de gestion des données, centralisés ou répartis.

\footnotetext{
36 http://biblio.wikia.com/wiki/SRU/SRW

37 www.dicodunet.com/definitions/normes/unicode.htm
} 
L'OPAC, catalogue en ligne permettant l'accès à partir du réseau TCP/IP, doit assurer le traitement de façon indépendante et de façon simultanée et partagée par tous les services des bibliothèques. Il faut bien concevoir le catalogue en ligne pour permettre l'accès à tous les fonds de leurs collections.

Avec l'OPAC, de plus en plus de bibliothécaires voient la nécessité d'offrir des interfaces au Web aussi attrayantes, esthétiquement sophistiquées, et faciles à utiliser pour capter les usagers qui se tournent actuellement vers l'Internet (pour chercher des informations $90 \%$ des collégiens des Etats Unis vont sur le net et $2 \%$ seulement vont vers la bibliothèque ${ }^{38}$ ) et ils se dirigent surtout vers les moteurs de recherche les plus connus $62 \%$ Google).

Les portails forment ces interfaces enjambant tous les types de médias et migrant vers la gestion électronique des ressources (ERM). L'implantation d'un portail ${ }^{39}$ constitue actuellement une composante essentielle des projets de numérisation des bibliothèques et des centres de documentation. Les principales composantes d'un portail devront faire partie des fonctionnalités des SIGB qui comportent les éléments suivants :

- Une fonction de publication de pages html et de mise à jour du portail;

- Mise à disposition de services d'informations « courantes » sur un portail : gestion d'événements, brèves, agenda, forum, ...

Ces services sont en standard dans le Content Management System. Le CMS ${ }^{40}$ est un outil qui remplit plusieurs grandes fonctions dans le cadre d'un portail : il est à la fois un outil de création de portail intégrant des informations « courantes », un outil de publication et un outil de « manipulation » de documents numériques (stockage, éventuellement possibilité de description...).

La gestion du contenu est désormais omniprésente à travers les interfaces Web, ce qui a permis aux bibliothèques d'évoluer vers les ressources virtuelles.

\section{L'interfaçage avec les fournisseurs de données}

Afin de récupérer les notices et les données des références importantes, surtout que celles-ci manquent dans les pays du Sud, l'interfaçage de récupération des données à partir des réservoirs bibliographiques est une nécessité. Récupérer des notices complètes à partir des réservoirs bibliographiques comme OCLC, RLIN, ou à partir des cédéroms comme celui de la BNF.

Avec des systèmes comme Apogée, récupérer des données en open access du Wikipédia devient une des tâches courantes d'un bibliothécaire du Sud capable de

\footnotetext{
38

OCLC. Perceptions of Libraries and Information Resources (2005) p. 1-17.

39 Des exemples d'OPACs intégrés et intensifiés avec les fonctionnalités portiques sont disponibles avec le « WebPAC innovateur pro »de SirsiDynix

40 Les CMS les plus connus sont SPIP et Typo3.
} 
profiter pleinement des ressources ouvertes et disponibles. La mise en œuvre d'outils Web de recherche bibliographique intégrables dans un portail.

Un moteur de recherche peut être employé dans le portail, les logiciels incluent les modules de recherche Web avec les systèmes de recherche traditionnels.

Avec l'OPAC et l'Internet, les bibliothèques expriment leur intérêt pour un outil d'accès multilingue, parce qu'elles sont persuadées que le multilinguisme pose un problème à leurs utilisateurs internes, en même temps qu' aux usagers internationaux d'autres pays se connectant à distance.

\section{Interfaces multilingues intégrées au sein des SIGB}

Le multilinguisme est désormais une caractéristique essentielle des bibliothèques modernes. D'ailleurs, la gestion de l'ensemble des caractères diacritiques et des caractères non latins de la norme UNICODE est devenue un critère de choix dans les cahiers des charges pour les SIGB, lors des taches techniques du stockage, de l'affichage, de l'indexation, de la recherche et de l'impression des données.

Le développement des interfaces multilingues intégrées au sein d'un système de gestion de bibliothèques était dicté par les besoins spécifiques du bibliothécaire. L'interface multilingue se présentait le plus souvent sous forme d'outils d'aide aux professionnels, telles que les listes contrôlés bilingues ou multilingues, les listes d'autorités contrôlées, les listes de vedettes matières et les thésauri. Maintenant les systèmes sont de plus en plus orientés vers l'usager pour qu'il puisse formuler une requête dans une langue et retrouver l'information dans une autre langue, voire en plusieurs langues.

Le recours aux techniques plus sophistiquées de la recherche multilingue simultanée permet l'exploitation de résultats pour que l'usager s'affranchisse des barrières linguistiques. Des enquêtes ${ }^{41}$ ont été effectuées auprès des utilisateurs pour mieux identifier le problème de l'usage de plus d'une langue dans une bibliothèque. Ces enquêtes avaient pour objectif d'analyser l'attitude des usagers vis-à-vis de l'OPAC qu'ils utilisent et de délimiter leurs besoins éventuels à un recours aux outils multilingues. Selon les résultats de cette enquête, la recherche dans un OPAC conventionnel requiert des efforts répétés de la part des utilisateurs qui, dans la plupart des cas, demeurent insatisfaits. Par conséquent, les bibliothèques ont exprimé leur intérêt pour un outil d'accès multilingue aux catalogues en ligne, qui peut améliorer la qualité des services rendus.

Le signalement succinct des expérimentations et de méthodes de repérage de l'information est nécessaire. Certaines se sont développées au moyen de techniques très complexes de l'intelligence artificielle.

41 Voir l'enquête du NORDINFO : PQNSQNEN-TUMAINEN. Monitoring Online Catalogues in the Nordic Technological University Libraries. Nordinfo Nytt, ${ }^{\circ} 4$, 1992, pp. 23-27. Voir aussi: C. Synellis. Translib: user survey, Report 1.1, Patras. www.wcl.ee.upatras.gr/ai/papers/michos3.pdf 
L'exposé des techniques suivantes ${ }^{42}$, n'est qu'une brève démonstration des exemples pour développer des modules, des interfaces en Open Source capables de gérer le multilinguisme : la recherche de l'information par croisement linguistique $(\text { RICL })^{43}$

Cette technique de recherche de l'information est une technique multilingue orientée Web faisant appel aux dictionnaires lisibles par ordinateur et à la traduction automatique. La technique RICL tend à évoluer afin d'atteindre un haut degré de compatibilité avec les architectures des systèmes de recherche utilisés par les moteurs de recherche du Web à volume élevé.

\section{La recherche de l'information translinguistique $(\text { RIT })^{44}$}

La recherche translinguistique de l'information est une méthode de recherche multilingue qui vise à aider l'usager à exploiter les ressources informationnelles dans plusieurs langues. Cette technique est, soit basée sur la traduction automatique, soit axée sur les méthodes statistiques de recherche de l'information.

Plusieurs interfaces multilingues intégrées au sein d'un système de gestion de bibliothèques, développées, dans un cadre expérimental, démontrent qu'on peut trouver des systèmes appropriés aux SID multilingues. Il faut citer à titre d'exemple le système baptisé TRANSLIB ${ }^{45}$ qui fonctionne dans un environnement Windows et supporte trois langues à savoir l' anglais, le grec et l'espagnol.$^{46}$ TRANSLIB est fait d'une intégration d'outils d'information multilingues performants tels qu'un dictionnaire bilingue, des tables de conversion, un lexique terminologique.

Ces techniques sont-t- elles suffisantes pour démontrer la faisabilité de ce type d'accès multilingue ? Loin s'en faut, car elles ne révèlent aucunement les problèmes potentiels pouvant surgir au cas où des langues non-européennes additionnelles seraient adoptées par les SIGB.

De plus ces technologies ne répondent que partiellement aux attentes des usagers, qui aspirent à des solutions plus performantes basées sur la traduction automati-

42 Cet exposé a repris les données d'une thèse de notre confère Khaled Habchi portant sur : le bilinguisme dans les systèmes d'informatisation de bibliothèques. Dirigée par Hubert Fondin, Université de Bordeaux III, 2007.

43 AlLJLAY Mohammed, FRIEDER Ophir. Effective Arabic-English Cross-Language Information Retrieval Via Machine-Readable Dictionaries and Machine Translation (2002). http://citeseer.ist. psu.edu/555942.html

44 Robert Frederiking, Teruko Mitamura, Eric Nyberg, Jaime Carbonel. Translingual Information Access. www.Citeseer.pdu.ist

45 Stephanos MICHOS, Efstathios STAMATOS, Nikos FAKOTAKIS. Supporting Multilinguality in Library Automation Systems Using all Tools. Applied Artificial Intelligence, Vol. 13, N ${ }^{\circ}$, octobre 1999, pp. 679-703

46 E. STAMATATOS et [al]. An advanced tool to for supporting multilingual access to library catalogues. Proc. MULSAIC'97 workshop. Ed. C.D. Spyroupolos, Nagoya, Japon, 1997. 
que des textes intégraux, qui n'a toujours pas encore atteint le niveau escompté pour remplacer la traduction humaine.

\section{Conclusion}

Les tendances actuelles doivent inclure les systèmes de repérage de l'information multilingue qui tiennent compte de la diversité linguistique et culturelle. En cela, les logiciels libres deviennent assez proches des principes et pratiques de la bibliothéconomie moderne. Les instances normatives documentaires prennent désormais en considération l'élément linguistique dans sa dimension universelle, et œuvrent à réviser l'ensemble des normes, procédures et protocoles dans ce sens.

Pour cela, il faut suivre de près les études et les recherches visant à développer des outils d'accès multilingue à l'information. Il faut avoir des cellules de veille pour filtrer les études pléthoriques qui couvrent des thématiques vastes telles que le multilinguisme dans les systèmes d'informatisation de bibliothèques, l'accès translinguistique à l'information, la recherche de l'information par croisement linguistique et l'indexation basée sur la traduction automatique, etc.

Cela est de nature à faciliter davantage le travail collaboratif entre tous les pays du monde, à favoriser l'échange des données, et encourager les bibliothèques du sud à se mettre à niveau.

Cependant il ne faut pas sous-estimer les obstacles: pour cela nous croyons en l'obligation de pratiquer une veille sur les nouvelles dynamiques d'accès aux sources et ressources offertes et profiter de la vague actuelle de l'ouverture (free, Open Access open sources) et d'y adhérer. Car très vite, cette mouvance sera récupérée, malgré les bonnes intentions, par le capitalisme financier et par les grandes multinationales.

Pour être optimiste, nous plaçons l'engagement dans le logiciel libre comme une résistance au capitalisme informationnel dont la capacité de détournement du savoir au service du pouvoir est de plus en plus active. 